\title{
Tumours of the anterior uvea. II. Intranuclear cytoplasmic inclusions in malignant melanoma of the iris
}

\author{
M. S. NOOR SUNBA, AMJAD H. S. RAHI, AND GWYN MORGAN \\ From the Department of Pathology, Institute of Ophthalmology, London
}

SUMMARY Intranuclear cytoplasmic inclusions have been described previously in both human and animal tissues. So far as we are aware they are described in detail for the first time in this paper in 60 cases of malignant melanoma of the iris, usually in spindle cells. The light microscopic appearances are described, and electron microscopic studies of selected cases show clearly the genesis of these inclusions by cytoplasmic invagination of the nuclear membrane. Their significance is discussed. It is of interest that we have seen identical inclusions in malignant melanomas of the ciliary body, choroid, and conjunctiva but have not as yet reported our findings.

Although nuclear inclusions have been described on a number of occasions, ${ }^{1-4}$ both in human and animal tissues, when they were assumed to be of viral origin, it was not until the introduction of the electron microscope that the largest group of nuclear inclusions were found to be cytoplasmic invaginations of the nuclear membrane. ${ }^{5}$ In an electron microscopic study of a case of tapioca melanoma of the iris ${ }^{6}$ curious intranuclear pigment inclusions were described. A brief reference has also been made to cytoplasmic invaginations of the nucleus in a case of iris melanoma, ${ }^{7}$ but the authors did not fully document the cytoplasmic inclusions that we are describing in the present paper, the purpose of which is to report in detail the incidence and morphology of these nuclear inclusions in malignant melanomas of the iris by both light and electron microscopy and to trace their genesis by nuclear invagination.

\section{Materials and methods}

During the light microscopic study of routine sections of 199 cases of malignant melanoma of the iris, stained by haematoxylin and eosin, nuclear inclusions were observed in 60 cases. Some of the latter were examined after staining by the periodic acid Schiff (PAS) method before and after diastase treatment, by methyl green-pyronin, by the Fontana stain, and with amino acid stains. In addition $1 \mu \mathrm{m}$ thick plastic sections of some cases were stained with

Correspondence to Dr A. H. S. Rahi, Institute of Ophthalmology, Judd Street London WC1H 9QS.

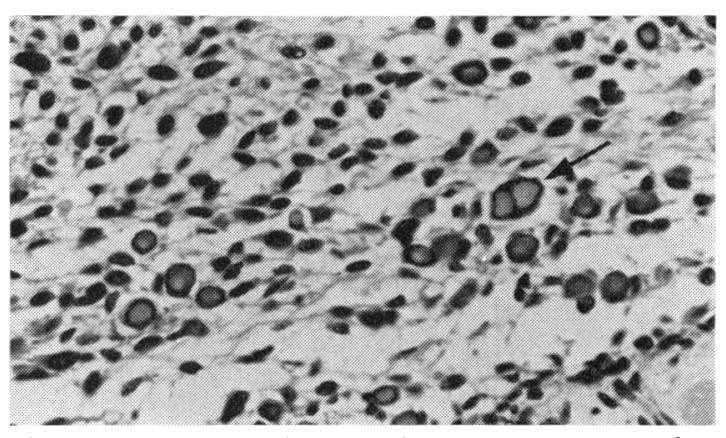

Fig. 1 Malignant melanoma of iris, showing intranuclear inclusions of varying size and shape. One nucleus (arrow) contains 2 inclusions. (Haematoxylin-eosin, $\times 245$.)

toluidine blue, and paraffin embedded sections of other melanomas were postfixed in osmium tetroxide for electron microscopic examination by the JEOL JEM-100C instrument. One malignant melanoma of the choroid was freshly fixed in glutaraldehyde and blocked in Araldite for similar studies.

\section{Results}

As stated in the previous paragraph, intranuclear inclusions were seen on light microscopy in 60 cases, 40 being females and 20 males. Fifty-seven of the tumours were spindle cell melanomas and 3 were of the mixed type. Inclusions were usually found in spindle cells. Some were small and others almost filled the nucleus; they varied in shape between spindle, spherical, and bizarre, and some nuclei contained two inclusions (Fig. 1). Some were situated 


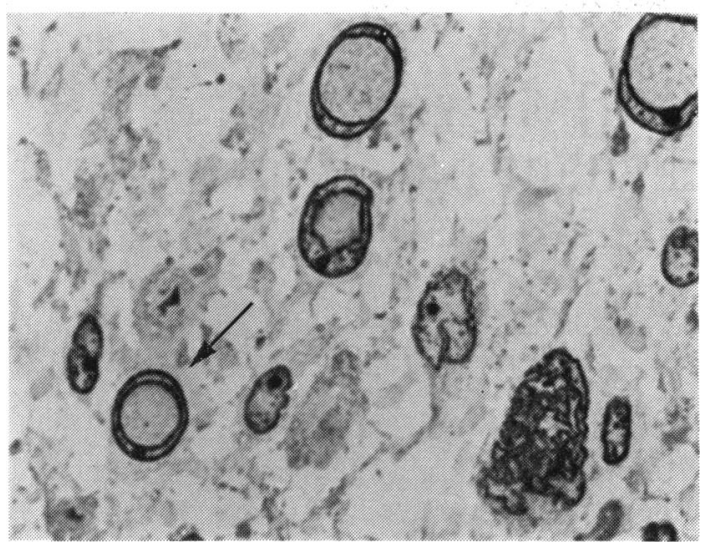

Fig. 2 Plastic section, showing centrally placed inclusion (arrow). (Toluidine blue, $\times 1440$.)

centrally in the nucleus (Fig. 2), and others were found at one pole, which was often distended. Not infrequently both the nucleus and cytoplasm become distended. The inclusions had a limiting membrane similar to the nuclear membrane and sometimes contained fine granules resembling melanin granules.

Inclusions stained with eosin with a varying degree of intensity and were PAS-positive both before and after diastase treatment. With the methyl green-pyronin stain both the inclusions and the cytoplasm of the cells stained red. With the Fontana stain a few inclusions contained faintly staining melanin granules. The inclusions stained positively with varying intensity for tyrosine and for the sulphur-containing amino acids (Table 1$){ }^{8}$

Plastic sections and electron microscopic sections showed clearly that the inclusions were of cytoplasmic origin and followed invagination of the nuclear membrane (Fig. 3). They contained cytoplasmic structures such as mitochondria, premelanosomes,

Table 1 Histochemical staining of intranuclear inclusions

\begin{tabular}{lll}
\hline Method (Pearse) & Result \\
\hline $1 \quad$ PAS & Positive \\
2 Diastase-PAS & Positive \\
$3 \quad$ Fontana & Positive \\
4 & Tyrosine (DC) & Positive \\
5 & Sulphur-containing & Positive \\
& amino acids: cystine, & \\
& cystein, methionine & \\
6 & Tryptophan (DMAB) & Negative \\
7 & Arginine (Sakaguchi) & Negative \\
8 & Methyl green-pyronin & Pyroninophilic \\
\hline
\end{tabular}

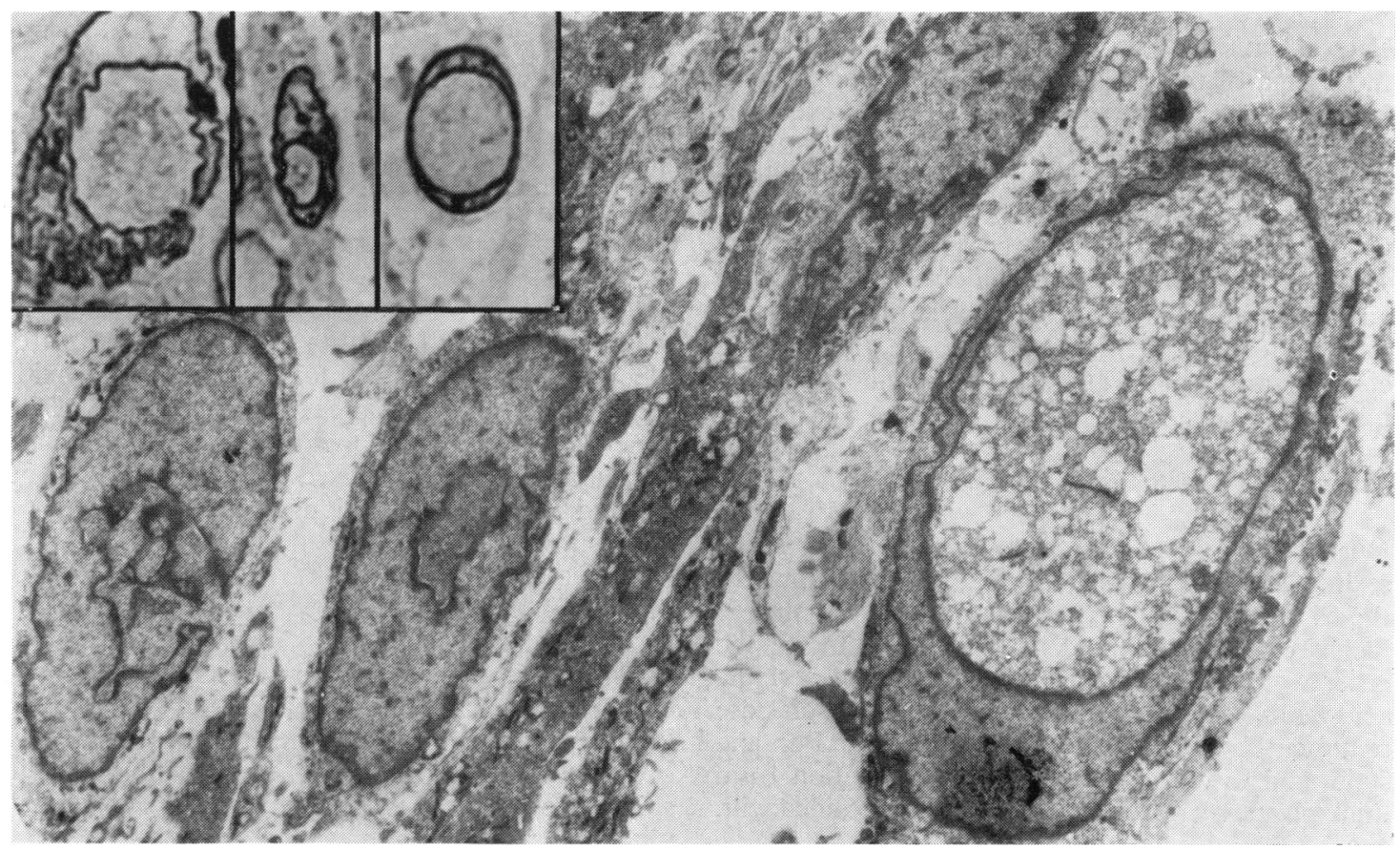

Fig. 3 Electron micrograph, showing the genesis of the inclusions by nuclear invagination $(\times 8750)$. Inset are plastic sections showing similar features. (Toluidine blue, $\times 1600$.) 


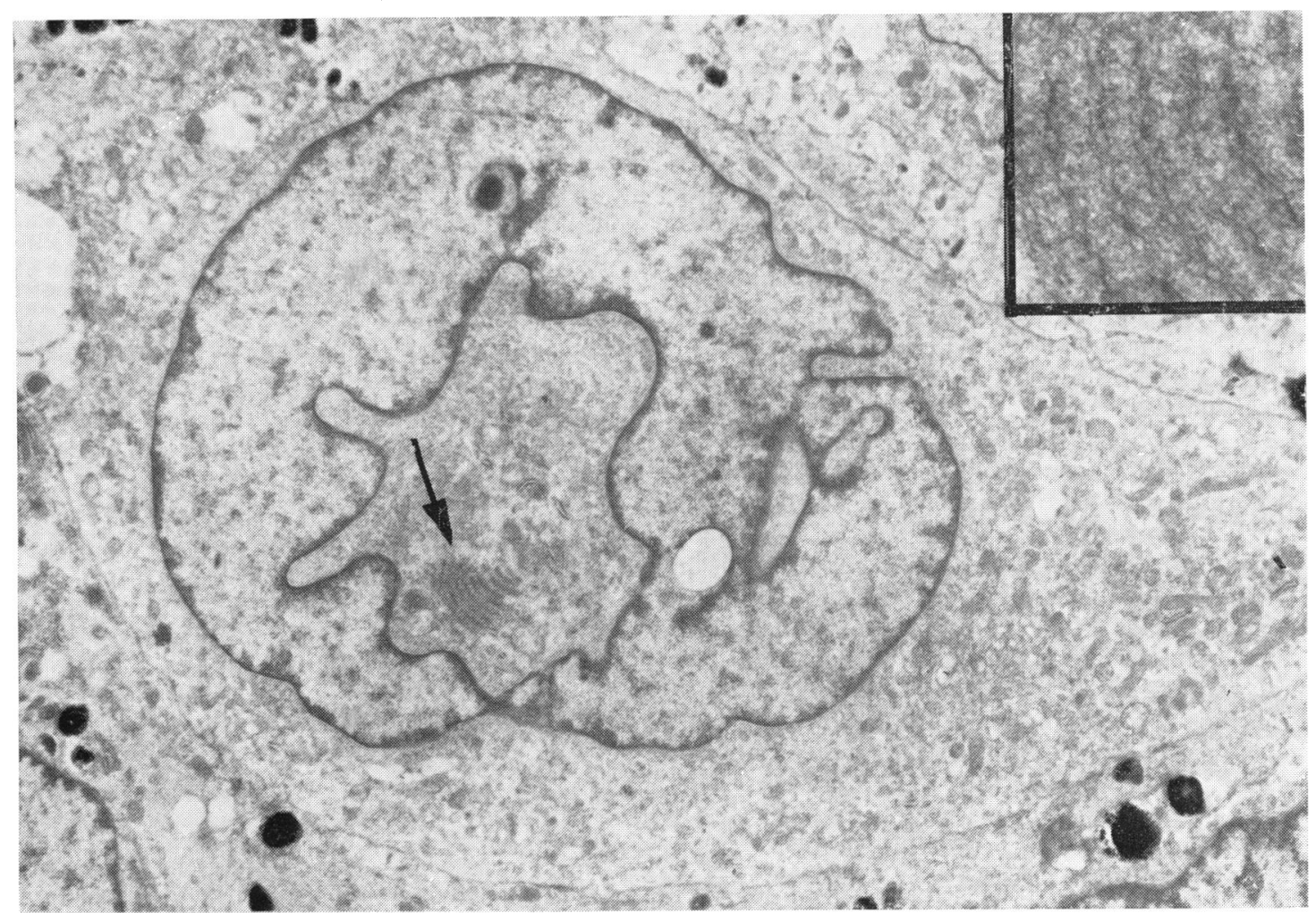

Fig. 4 Electron micrograph, showing an inclusion which contains annulate lamellae (arrow) $(\times 13680)$ which are greatly magnified in the inset $(\times 30580)$.

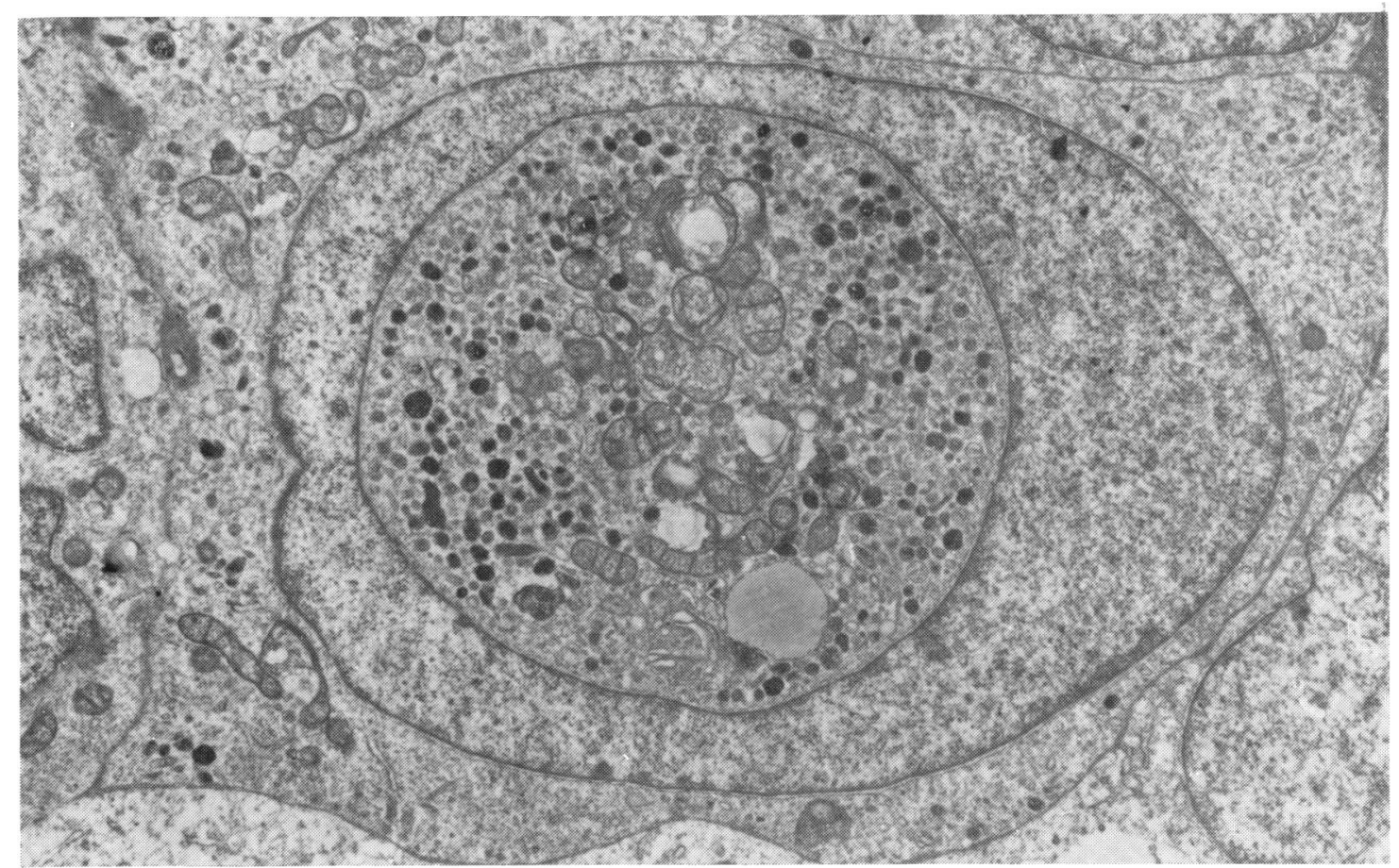

Fig. 5 Electron micrograph of a malignant melanoma of the choroid, showing a nuclear inclusion in which there are well preserved mitochondria, premelanosomes, and melanosomes $(\times 8960)$. 
melanosomes, microfilaments, ribosomes, rough endoplasmic reticulum, and annulate lamellae (Fig. 4), and were separated from the nuclear matrix by a double-layered membrane, the outer aspect of which consisted of condensed chromatin similar to that of the inner aspect of the nucleus. The inner aspect of the membrane was studded with ribosomes as is the outer aspect of the nuclear envelope. Electron microscopy of a malignant melanoma of the choroid showed well preserved mitochondria, premelanosomes, and melanosomes (Fig. 5).

\section{Discussion}

Although cytoplasmic intranuclear inclusions similar to those described in this paper have been reported previously, ${ }^{7-13}$ to our knowledge this is the first occasion that they have been described in detail in malignant melanomas of the iris. We should like to add that we have also seen very similar inclusions in malignant melanomas of the ciliary body, choroid, and conjunctiva.

The intranuclear inclusions found in malignant melanomas of the iris varied in size, shape, site, and density of staining. Although we did not carry out histochemical studies for enzymes, which are found exclusively in the cytoplasm, electron microscopy showed quite clearly that they were cytoplasmic invaginations of the nuclear membrane, so that in effect they are pseudoinclusions rather than true inclusions.

The genesis of the nuclear invagination is unknown. Some authors ${ }^{14}$ found the inclusions in large cells which contained much cytoplasm. Leduc and Wilson ${ }^{5}$ consider that the invagination develops as an attempt to maintain the normal ratio of nuclear surface to nuclear volume, while Sobel et al. ${ }^{15}$ consider that the majority of inclusions can be explained by the swollen cytoplasm extruding into the nucleus. Serber ${ }^{10}$ studied the development of cytoplasmic intranuclear inclusions in the pituitary gland of experimental animals and found that they were related to the process of aging and probably developed due to a deficiency of sex hormones.

Although we do not understand the genesis of these intranuclear cytoplasmic inclusions, we think that there may be an increase in the effective nuclear surface and the associated cytoplasm which leads to the invagination of the nuclear membrane by the cytoplasm. The significance of the annulate lamellae in some inclusions is not clear, but similar structures in the cytoplasm of normal epithelium have been identified with active protein synthesis. ${ }^{16}$ It is tempting to postulate, therefore, that these struc- tures play a similar role in tumour cells. An active protein synthesis with consequent increase in cytoplasmic volume could lead to the cytoplasmic invagination of the nuclear membrane. Because of the finding of inclusions of different shape and size in sections of the same tumour, it is reasonable to conclude that there is a continuous process producing the inclusions.

It is interesting that 40 of our patients were females, for the prognosis from metastases of malignant melanoma of the iris is better in females. ${ }^{17}$ Is it possible, therefore, that these inclusions could be a pointer to a better prognosis in iris melanomas?

\section{References}

${ }^{1}$ Cowdry EV. The problem of intranuclear inclusions in virus diseases. Arch Pathol 1924; 18 : 527-42.

${ }^{2}$ Fischmann CF, Russell DS. The occurrence of intranuclear inclusions in culture of foetal leptomeninges. $J$ Pathol Bacteriol 1940; 50: 53-9.

${ }^{3}$ Zollinger HU. Beitrag zur pathogenese der einschlusskörper. Schweiz Z. Allg Pathol 1951; 14: 446-55.

${ }^{4}$ Kleinfeld R, Koulish S. Cytological aspects of mouse and rat liver containing nuclear inclusions. Anat Rec 1957; 128: 443-5.

${ }^{5}$ Leduc EH, Wilson JW. An electron microscopic study of intranuclear inclusions in mouse liver and hepatomas. J Biophys Biochem Cytol 1959; 6: 427-30.

${ }^{6}$ Iwamoto T, Reese AB, Mund ML. Tapioca melanoma of the iris. Part 2. Electron microscopy of the melanoma cells compared with normal iris melanocytes. Am J Ophthalmol 1972; 74: 851-61.

${ }^{7}$ Lommatzsch P, Klug $\mathbf{H}$. Ein beitrag zur ultrastrukur des irismelanoms. Albrecht von Graefes Arch Klin Ophthalmol 1977; 203: 101-17.

${ }^{8}$ Pearse AGE. Histochemistry, Theoretical and Applied, 3rd ed. London: Churchill 1968.

${ }^{9}$ Leduc EH, Wilson JW. A histochemical study of intranuclear inclusions in mouse liver and hepatoma. $J$ Histochem Cytochem 1959; 7: 8-16.

${ }^{10}$ Serber BJ. Large nuclear inclusions in pituitary gland basophils of golden hamster. Anat Rec 1961; 139: 345-52.

"Bouteille M, Kalifat SR, Delarue J. Ultrastructural variations of nuclear bodies in human disease. $J$ Ultrastruct Res 1967; 19: 474-86.

${ }^{12}$ Sorbino-Simóes MA, Conçlaves V. Nuclear bodies in papillary carcinomas of the human thyroid gland. Arch Pathol 1974; 98 : 94-9.

${ }^{13}$ Ghadially FN. Ultrastructural Pathology of the Cell. London: Butterworths 1975: 50-78.

${ }^{14}$ Wessel W. Elektronemikroskopische untersuchungen von intranucleären einschlusskörpern. Virchows Arch (Pathol Anat) 1958; 331: 314-28.

${ }^{15}$ Sobel HJ, Schwartz R, Marquet MS, Passaic NJ. Non-viral nuclear inclusions. I. Cytoplasmic invaginations. Arch Pathol 1969; 87: 179-92.

${ }^{16}$ Hogan MJ, Alvarado JA, Weddell JE. Histology of the Human Eye. An Atlas and Text Book. Philadelphia: Saunders, 1971: 415.

${ }^{17}$ Sunba MSN, Rahi AHS, Morgan G. Tumours of the anterior uvea: I. Metastasizing malignant melanoma of the iris. Arch Ophthalmol 1980; 98: 82-5. 